\title{
Correlation of Optic Nerve Sheath Diameter with Direct Measurement of Intracranial Pressure through an External Ventricular Drain
}

\author{
M Asghar Ali $^{1}$, Madiha Hashmi ${ }^{1}$, Shahzad Shamim ${ }^{2}$, Basit Salam ${ }^{3}$, Sheema Siraj ${ }^{1}$, Bushra Salim ${ }^{1}$ \\ 1. Anaesthesiology, Aga Khan University Hospital, Karachi, PAK 2. Section of Neurosurgery, Aga Khan University \\ Hospital, Karachi, PAK 3. Radiology, Aga Khan University Hospital, Karachi, PAK
}

Corresponding author: M Asghar Ali, asghar.ashraf@aku.edu

\section{Abstract \\ Background}

Early diagnosis and management of raised intracranial pressure (ICP) is essential for preventing brain damage and even death. Invasive monitoring is the gold standard to measure raised ICP but it may not be feasible in a heterogeneous group of patients. Noninvasively, a simple bedside ocular ultrasound can detect elevated ICP. The aim of our study was to evaluate the correlation between optic nerve sheath diameter (ONSD) and direct ICP measurements and to determine sensitivity and specificity of ONSD measurements to detect elevated ICP $\left(>15 \mathrm{~cm} \mathrm{H}_{2} \mathrm{O}\right)$.

\section{Methods}

This prospective study was conducted at the intensive care unit/high dependency units/wards of Aga Khan University Hospital. Patients with external ventricular drain (EVD) for intracranial hypertension were enrolled. Ocular ultrasound was performed with a $7.5 \mathrm{MHz}$ linear probe. For each subject, three measurements on each eye were performed and the mean of the six measurements was determined. EVD was temporarily occluded and the ICP was recorded every minute for five minutes. A receiver operative characteristics (ROC) curve was constructed to determine the optimal ONSD cutoff to detect ICP above 15 $\mathrm{cm} \mathrm{H}_{2} \mathrm{O}$.

\section{Results}

A total of 35 adult patients were included in this study. The ONSD was linearly correlated with ICP in both right and left eyes $(r=0.662, p=0.0005$ and $r=0.449 ; \mathrm{p}<0.002)$ respectively. Pearson correlation of ONSD between two eyes (right and left) was $0.749 ; p=0.0005$ and $0.726 ; p=0.005$ at day 1 and day 2 , respectively. ROC curve was created and observed that AUC of right and left eyes was 0.815 (95\% CI: 0.61 to 0.99 ) and 0.69 ( $95 \%$ CI: 0.37 to 0.99 ).

Received 07/09/2019

Review began 07/21/2019 Review ended 09/24/2019 Published 09/26/2019

\section{(c) Copyright 2019}

Ali et al. This is an open access article distributed under the terms of the Creative Commons Attribution License CC-BY 3.0., which permits unrestricted use, distribution, and reproduction in any medium, provided the original author and source are credited.

\section{Conclusion}

According to this study, ventriculostomy measurements of ICP are directly correlated with ultrasound ONSD measurements. Hence, we conclude that ONSD measured by ocular ultrasound is a simple yet effective method to detect raised ICP.

Categories: Anesthesiology, Radiology, Neurosurgery

Keywords: optic nerve sheath diameter, ocular ultrasound, intracranial, intracranial pressure (icp)

\section{Introduction}

Raised intracranial pressure (ICP) is a common manifestation of severe brain injury that requires rapid diagnosis and therapeutic intervention to prevent possible brain damage or death. But the diagnosis of elevated ICP requires expertise. Recommendations are to keep ICP $<20-25 \mathrm{mmHg}$ in the setting of traumatic brain injury (TBI) as well as other forms of acute brain injury [1,2]. Invasive ICP monitoring, with either an intra-parenchymal probe or an intra-ventricular catheter, is associated with certain risks, for example, hemorrhage and infection [3,4]. Computed tomography (CT) and magnetic resonance (MR) images, showing effacement of basal cisterns, diffuse sulci effacement and the presence of significant midline shift, are often used to make decisions on the management of intracranial hypertension, although the accuracy is still unclear. An accurate and reliable noninvasive tool to detect the presence of intracranial hypertension would be of significant value, not only in situations where there is clinical suspicion for intracranial hypertension but invasive monitoring is unavailable or associated with possible risks. Also, high risk candidates for invasive monitoring can be screened out using a simple noninvasive tool.

Intracranial pressure can be definitively measured and monitored through placement of invasive monitoring 
devices such as an external ventricular drain (EVD) [5, 6]. This involves inserting a fine tube inside the lateral ventricle through a small burr hole, and direct measurement of cerebrospinal fluid (CSF) pressure from within the ventricle. Besides gold standard, continuous ICP monitoring EVD also allows intermittent drainage of CSF, thus reducing ICP. Noninvasively, a simple measurement of ocular ultrasound (US) is an emerging technique to detect elevated ICP at the bedside. The ultrasonographic measurement of the optic nerve sheath diameter (ONSD) has been evaluated as a non-invasive method to identify the presence of raised ICP, in patients with TBI and intracranial hemorrhage (ICH) [7-10]. ONSD has been correlated with clinical symptoms and CT abnormalities in many clinical studies but the association with direct measurement from EVD has only been examined in one study [11].

The aim of our study was to evaluate the correlation between ONSD and direct ICP measurements and to determine sensitivity and specificity of ONSD measurements to detect elevated ICP (>15 cm H2O).

\section{Materials And Methods}

This prospective study was conducted at the intensive care unit/high dependency units/wards of Aga Khan University Hospital, after approval from the hospital ethics committee. Patients with EVD for intracranial hypertension were enrolled and written informed consent was taken from next of kin of all the patients. All adult patients admitted to the intensive care unit/high dependency units/wards, who already had an EVD in place, were included while patient's or family's refusal to participate in the study, known orbital injury and pre-existing optic nerve pathology were excluded.

Both ICP and US measurements were done on days 1 and 2 during intracranial pressure monitoring by the primary investigator. ICP was measured manually in $\mathrm{mmHg}$, using the rise of CSF in the water-column against the zero reference point on patients' external auditory canal, using the commercially available closed CSF drainage system. All ONSD scans were performed using Mindray sonosite ultrasound machine with a $7.5 \mathrm{MHz}$ linear array probe with orbital imaging settings and a high resolution optimization setting. The scan was done by placing the probe on the superior and lateral aspect of the orbit against the upper eyelid with the eye close and angle slightly caudally and medially until the optic nerve was visualized as a linear hypo echoic structure with clearly defined margins posterior to the globe. For each subject, three measurements on each eye were performed and the mean of the six measurements was determined to yield a mean ONSD. During sonography, EVD was temporarily occluded and the ICP was recorded every minute for five minutes.

\section{Data analysis procedure}

Sample size calculation was performed by using PASS software 11.0.4 (NCSS, LLC, Kaysville, UT). A sample size was calculated to be 35 patients needed to achieve $90 \%$ power to observe the correlation of ONSD and ICP was 0.59 using a two-sided hypothesis test with 0.01 significance level. All statistical analysis was performed using Statistical Packages for Social Science version 19 (SPSS Inc., Chicago, IL). Age, weight, height, ICP and ONSD were quantitative variables so Kolmogorov-Smirnov (K-S) tests were used for normality. Mean with standard deviation or median (IQR) was computed for age, weight and height and ONSD. Scatter plot and linear regression were used to assess the relationship between ONSD and ICP. Partial correlation analysis was performed to observe the effect of covariate (age, BMI). A receiver operative characteristics (ROC) curve was constructed to determine the optimal ONSD cutoff to detect ICP above 15 cm H2O. $\mathrm{P} \leqslant 0.05$ was considered significant.

\section{Results}

A total of 35 adult patients admitted to the intensive care unit/high dependency units/wards were included in this study. Mean age of all patients was $40.60 \pm 14.53$ years (range: $20-70$ ). The detailed information on the demographic and surgery of the patients is given in Table 1. At day 1, mean ICP was $16.34 \pm 1.07 \mathrm{mmHg}$ [95\% CI: 15.97 to 16.71 ] and ONSD for right and left eyes was $7.07 \pm 0.41 \mathrm{~mm}$ [95\% CI: 6.94 to 7.21 ], and 7.14 $\pm 0.38 \mathrm{~mm}[95 \% \mathrm{CI}: 7.01$ to 7.27$]$ respectively. The ONSD was linearly correlated with ICP in both right and left eyes $[\mathrm{r}=0.662, \mathrm{p}=0.0005$ and $\mathrm{r}=0.449 ; \mathrm{p}<0.002]$ respectively as shown in Figure 1 . At day 2 , mean ICP was $11.18 \pm 1.23 \mathrm{mmHg}$ [95\% CI: 10.75 to 11.61$]$ and ONSD for right and left eyes was $4.89 \pm 0.41 \mathrm{~mm}[95 \%$ CI: 4.74 to 5.04 ] and $4.88 \pm 0.37 \mathrm{~mm}$ [95\% CI: 4.75 to 5.01$]$, respectively. The ONSD was moderately correlated with ICP in both right and left eyes $[\mathrm{r}=0.387, \mathrm{p}=0.0022$ and $\mathrm{r}=0.544 ; \mathrm{p}<0.001]$ respectively as shown in Figure 2. Pearson correlation of ONSD between two eyes (right and left) was $0.749 ; \mathrm{p}=0.0005$ and $0.726 ; \mathrm{p}=0.005$ at day 1 and day 2 , respectively. The partial correlation analysis was performed and observed that there was no effect of covariate (Age and BMI) on correlation between ONSD and ICD as shown in Table 2. For day 1, ICP of 4 (11.4\%) cases was observed $\leqslant 15 \mathrm{~mm}$ (Normal) and 31 (88.6\%) had more than $15 \mathrm{~mm}$ (raised). ROC curve was created and observed that AUC of right and left eyes was 0.815 [95\% CI: 0.61 to 0.99 ] and 0.69 [95\% CI: 0.37 to 0.99 ] (Figure 3). 


\section{Cureus}

\section{Variables}

Age (Years)

Weight (kg)

Height (cm)

BMI $\left(\mathrm{kg} / \mathrm{m}^{2}\right)$

Surgery

Craniotomy

Decompressive craniotomy

EVD insertion only

Mini-craniotomy for excision of cyst
Point Estimates

$40.60 \pm 14.53$

$75.71 \pm 9.74$

$170.71 \pm 12.03$

$26.17 \pm 4.12$

$11(31.5 \%)$

$4(11.4 \%)$

$19(54.3 \%)$

$1(2.9 \%)$

TABLE 1: Characteristics of study participants $(n=35)$.

EVD: External ventricular drain

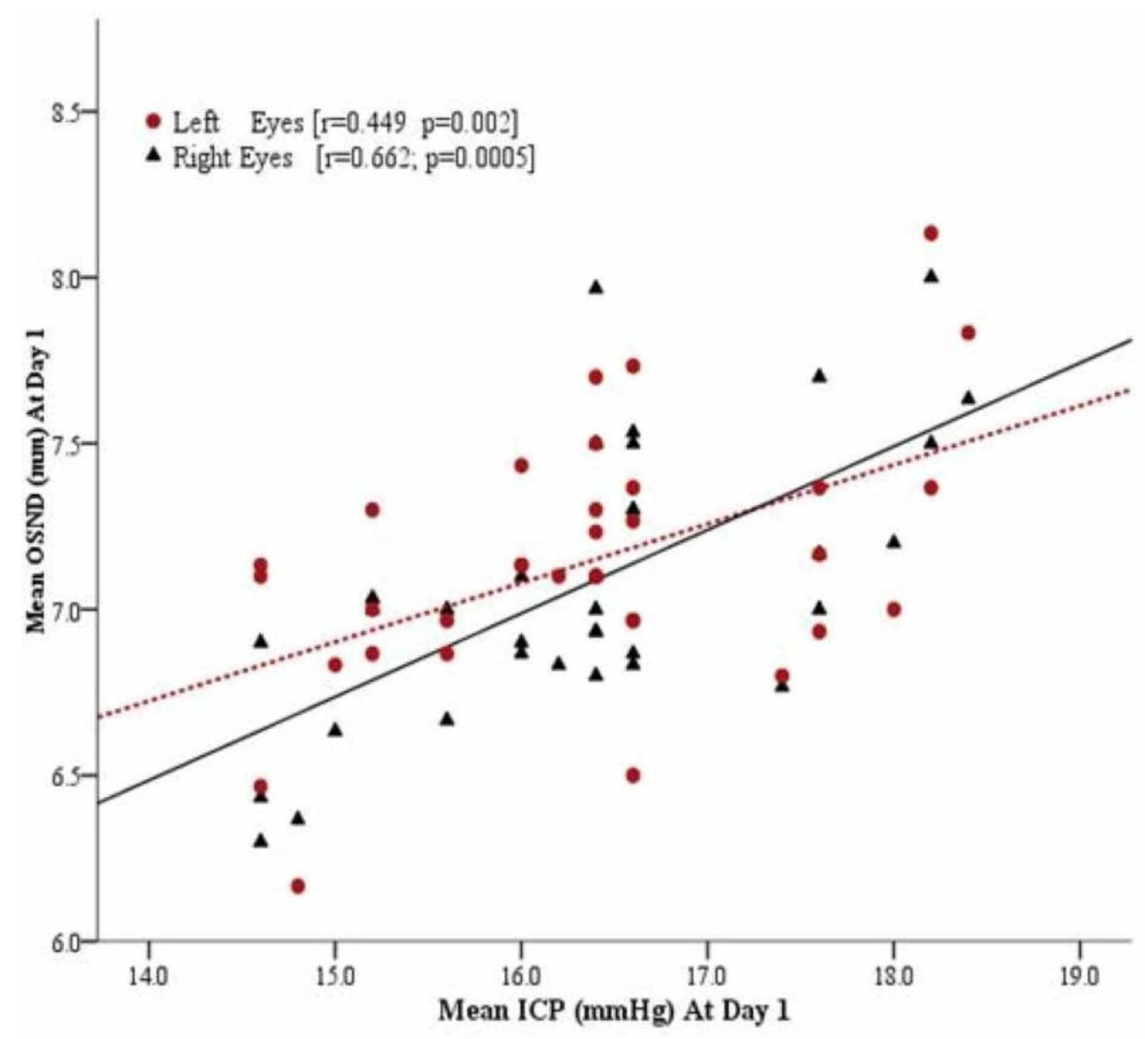

FIGURE 1: Scatter plot showing the relationship between the ONSD and ICP at day $1(n=35)$. The linear prediction from regression is shown as solid or dotted line.

ONSD: Optic nerve sheath diameter; ICP: Intracranial pressure. 


\section{Cureus}

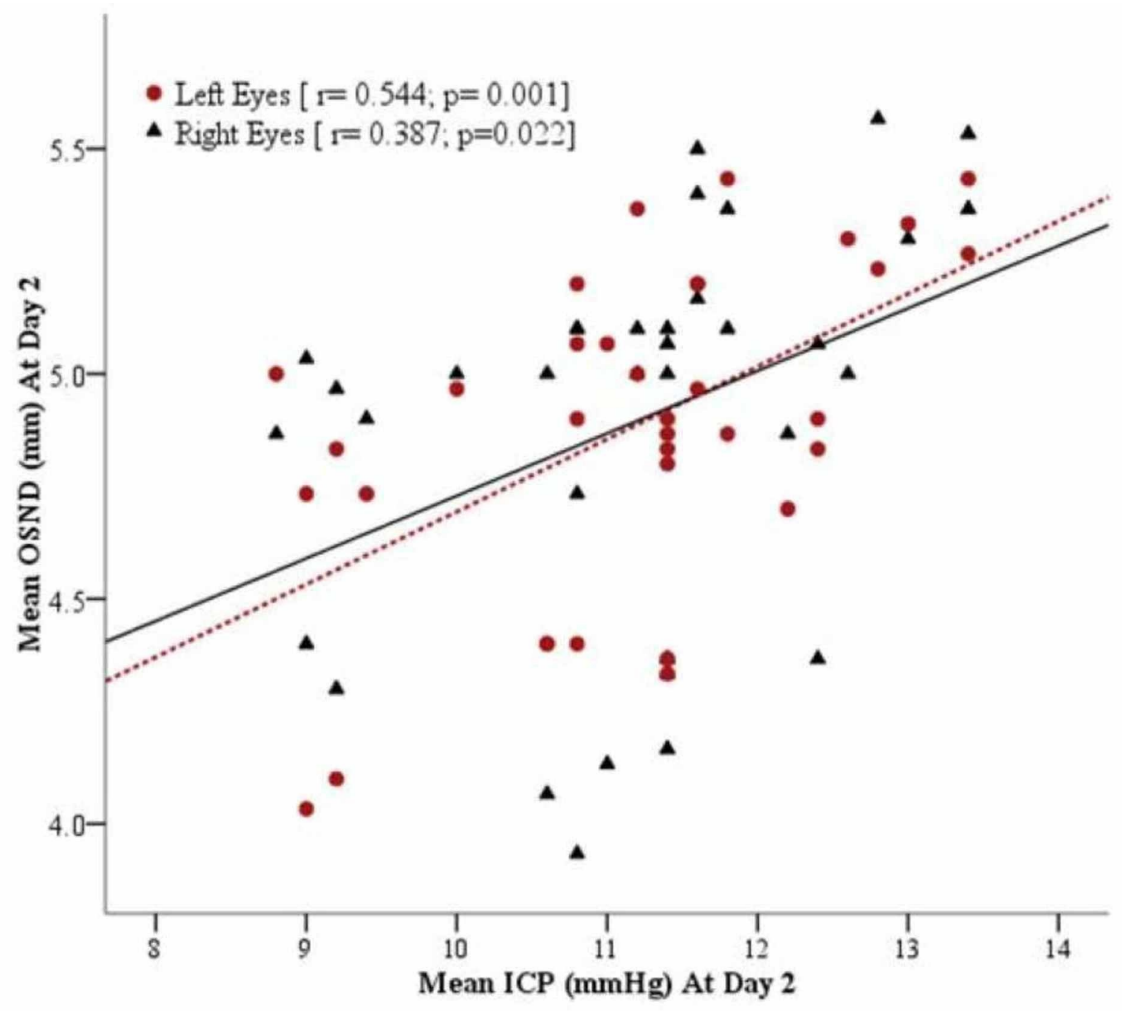

FIGURE 2: Scatter plot showing the relationship between the ONSD and ICP at day $2(n=35)$. The linear prediction from regression is shown as solid or dotted line.

ONSD: Optic nerve sheath diameter; ICP: Intracranial pressure.

\begin{tabular}{|c|c|c|c|c|c|}
\hline \multirow{2}{*}{ Control Variables } & \multirow{2}{*}{ Value } & \multicolumn{2}{|l|}{ Day 1} & \multicolumn{2}{|l|}{ Day 2} \\
\hline & & Right Eye & Left Eye & Right Eye & Left Eye \\
\hline \multirow{2}{*}{ Age (Years) } & $r$ & 0.663 & 0.497 & 0.384 & 0.542 \\
\hline & P-Value & 0.0005 & 0.003 & 0.025 & 0.001 \\
\hline \multirow{2}{*}{ BMI $\left(\mathrm{kg} / \mathrm{m}^{2}\right)$} & $r$ & 0.655 & 0.484 & 0.409 & 0.546 \\
\hline & P-Value & 0.0005 & 0.004 & 0.016 & 0.001 \\
\hline
\end{tabular}

TABLE 2: Partial correlations between the ONSD and ICP after controlling covariates.

ONSD: Optic nerve sheath diameter; ICP: Intracranial pressure. 


\section{Cureus}

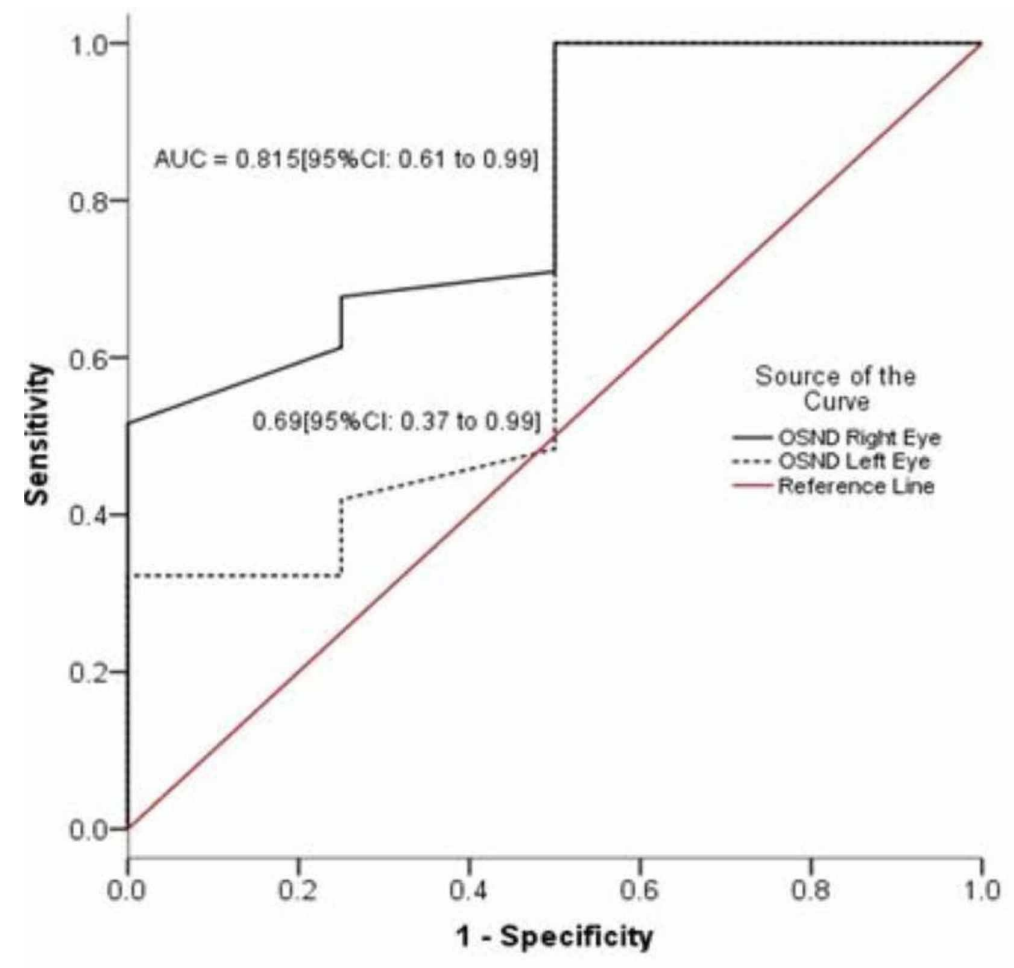

FIGURE 3: Receiver operating characteristic curve for ONSD of right and left eye.

ONSD: Optic nerve sheath diameter

\section{Discussion}

Raised ICP is a dreaded complication of neurological diseases that often leads to adverse outcomes. There is increasing evidence that ICP monitoring improves outcomes [12]. Clinical signs of raised ICP are often unreliable or too late to manifest and may lead to unacceptable delay in therapeutic intervention. Invasive measurement by an intraventricular or intraparenchymal catheter is the gold standard and used extensively in the management of traumatic brain injury; however, it may not be feasible in a heterogenous group of medical patients [13].

Non-invasive tests such as transcranial Doppler (TCD), tympanic membrane displacement (TMD), and US have been used to detect raised ICP. The percentage of unsuccessful measurements is very high, around 60\% with TCD and TMD. Moreover, TMD testing is time consuming [11]. Hence, these non-invasive methods can be impractical in urgent situations such as ED or ICU [14]. On the other hand, US measurement of ONSD is reproducible, efficient and easily learned technique without a steep learning curve and has low intra- and interobserver variation [11].

There is a well-established role of ultrasographic measurement of optic nerve diameter in literature. Optic nerve sheath diameter is directly related to the raise in ICP through the subarachnoid space. Raised ICP leads to distention of the optic nerve sheath without any delay leading to early detection of acute elevation in ICP [15]. There has been considerable disagreement on the normal limit of ONSD in literature, and the ONSD range varies in studies done on healthy volunteers [16-19]. We did a prior study to evaluate the upper limit of normal ONSD in Pakistani population and found it to be $4.82 \mathrm{~mm}$ [20].

In this study, a significant correlation was found between raised ICP and ONSD measured by ocular ultrasound, which is also supported by other studies. ICP measured through EVD and ONSD values obtained on admission were strongly correlated. Moreover, ultrasonographic ONSD measurements were successfully used to determine the changes in ICP that occurred during first and second postoperative day. Jeon et al. also found significant correlation of ultrasonographic measurement of ONSD and directly measured ICP using an EVD catheter $(r=0.77, \mathrm{p}<0.01)$.

Although the dilation of optic nerve sheath is directly related to increase in ICP as reported in many studies whether the ONSD would decrease after the ICP decreased in vivo is still unclear. Hansen et al. demonstrated the changes in ONSD, in vitro by the application of incremental and decreased pressure steps in the 
subarachnoid space [21]. However, there are few clinical studies that reported the ONSD variations following treatment for elevated ICP. Launey et al. reported a significant correlation between the ICP and ONSD measurements obtained before and after mannitol infusion [22]. In our study also, the diameter of optic nerve sheath decreased after the treatment to reduce ICP. This pressure dependent behavior of the optic nerve sheath may be due to its exceptional elastic properties. According to Killer et al., there are various trabeculae, septa and stout pillars in the subarachnoid space of the human optic nerve [23]. Therefore, these trabeculae stretch when ICP is increased thereby dilating the optic nerve sheath. Similarly causing the optic nerve diameter to decrease upon reduction of ICP due to refolding of these trabeculae, due to the elasticity of the optic nerve sheath. Hence ONCD examinations can be used to detect the variations in the ICP dynamically.

Accuracy of US measurement of ONSD has been compared with MRI measurements. Bäuerle et al. compared US and MRI for ONSD. According to them US is a noninvasive bedside tool for longitudinal ONSD measurements [24]. Shirodkar et al. also have correlated sonographic ONSD with the ONSD measured by MRI, concluding that US is as good as MRI [25].

Like all other techniques, ocular ultrasound has a learner's curve and the investigator may require practice to obtain reliable and reproducible image and to be able to differentiate the artifacts. To minimize the interobserver and intra-observer variability all scans were performed by a single investigator who had experience of scanning more than 100 healthy volunteers. A total of six readings were recorded and the mean was taken.

\section{Limitations}

This study has few limitations. Firstly, the sample size was modest. We recommend further studies with larger sample size in future so that the findings of this study can be validated. Secondly, all measurements were performed by the primary investigator which could lead to observer bias to the readings. Moreover, since all measurements were done by the primary investigator, the inter-observer variability could not be assessed in this study.

\section{Conclusions}

We conclude that ultrasound measurement of optic nerve sheath is a simple tool for detection of raised ICP. Further studies, with a larger sample size, are needed to validate our findings so that this modality can be used in clinical practice. Furthermore, ONSD should not be considered in isolation, it should be a part of a holistic approach, taking into consideration patient's clinical condition.

\section{Additional Information \\ Disclosures}

Human subjects: Consent was obtained by all participants in this study. Ethical Review Committee, Aga Khan University issued approval 3211-Ana-ERC-14. The study was reviewed and discussed at Aga Khan University. There was no major ethical issues. The study was given an approval. Animal subjects: All authors have confirmed that this study did not involve animal subjects or tissue. Conflicts of interest: In compliance with the ICMJE uniform disclosure form, all authors declare the following: Payment/services info: All authors have declared that no financial support was received from any organization for the submitted work. Financial relationships: All authors have declared that they have no financial relationships at present or within the previous three years with any organizations that might have an interest in the submitted work. Other relationships: All authors have declared that there are no other relationships or activities that could appear to have influenced the submitted work.

\section{References}

1. Bratton SL, Chestnut RM, Ghajar J, et al.: Cerebral perfusion thresholds. J Neurotrauma. 2007, 24:59-64. 10.1089/neu.2007.9987

2. Morgenstern LB, HemphillIII JC, Anderson C, et al.: Guidelines for the management of spontaneous intracerebral hemorrhage: a guideline for healthcare professionals from the American Heart Association/American Stroke Association. Stroke. 2010, 41:2108-2129. 10.1161/STR.0b013e3181ec611b

3. Ngo QN, Ranger A, Singh RN, Kornecki A, Seabrook J, Seabrook J, Fraser D: External ventricular drains in pediatric patients. Pediatr Crit Care Med. 2009, 10:346-351. 10.1097/PCC.0b013e3181a320cd

4. Ross IB, Dhillon GS: Ventriculostomy-related cerebral hemorrhages after endovascular aneurysm treatment AJNR Am J Neuroradiol. 2003, 24:1528-1531.

5. Helmke K, Hansen HC: Fundamentals of transorbital sonographic evaluation of optic nerve sheath expansion under intracranial hypertension. I. Experimental study. Pediatr Radiol. 1996, 26:701-705. 10.1007/bf01383383

6. Mayer S, Chong JY: Critical care management of increased intracranial pressure . J Intensive Care Med. 2002, 17:55-67. 10.1046/j.1525-1489.2002.17201.x

7. The Brain Trauma Foundation. The American Association of Neurological Surgeons. The Joint Section on Neurotrauma and Critical Care. Indications for intracranial pressure monitoring. J Neurotrauma. 2000, 17:479-491. 10.1089/neu.2000.17.479

8. Geeraerts T, Launey Y, Martin L, Pottecher J, Vigué B, Duranteau J, Benhamou D: Ultrasonography of the 
optic nerve sheath may be useful for detecting raised intracranial pressure after severe brain injury. Intensive Care Med. 2007, 33:1704-1711. 10.1007/s00134-007-0797-6

9. Moretti R, Pizzi B: Optic nerve ultrasound for detection of intracranial hypertension in intracranial hemorrhage patients: confirmation of previous findings in a different patient population. J Neurosurg Anesthesiol. 2009, 21:16-20. 10.1097/ANA.0b013e318185996a

10. Moretti R, Pizzi B, Cassini F, Vivaldi N: Reliability of optic nerve ultrasound for the evaluation of patients with spontaneous intracranial hemorrhage. Neurocrit Care. 2009, 11:406-410. 10.1007/s12028-009-9250-8

11. Jeon JP, Lee SU, Kim SE, et al.: Correlation of optic nerve sheath diameter with directly measured intracranial pressure in Korean adults using bedside ultrasonography. PloS One. 2017, 12:0183170. 10.1371/journal.pone.0183170

12. Chacko J: Optic nerve sheath diameter: an ultrasonographic window to view raised intracranial pressure? . Indian J Crit Care Med. 2014, 18:707-708. 10.4103/0972-5229.144007

13. Lele A, Kannan N, Vavilala MS, et al.: Patients who benefit from intracranial pressure monitoring without cerebrospinal fluid drainage after severe traumatic brain injury. Neurosurgery. 2019, 85:231-239. 10.1093/neuros/nyy247

14. Khan MN, Shallwani H, Khan MU, Shamim MS: Noninvasive monitoring intracranial pressure-A review of available modalities. Surg Neurol Int. 2017, 8:51. 10.4103/sni.sni_403_16

15. Bala R, Banerjee A: A study on ultrasonographic measurement of optic nerve sheath diameter for detecting raised intracranial pressure in intensive care unit patients. Anaesth Pain Intensive Care. 2017, 21:432-437.

16. Maude RR, Hossain MA, Hassan MU, et al.: Transorbital sonographic evaluation of normal optic nerve sheath diameter in healthy volunteers in Bangladesh. PloS One. 2013, 8:81013. 10.1371/annotation/4e2f88bd-e836-4e0c-a3e0-f6062331702b

17. Vaiman M, Gottlieb P, Bekerman I: Quantitative relations between the eyeball, the optic nerve, and the optic canal important for intracranial pressure monitoring. Head Face Med. 2014, 10:32. 10.1186/1746160X-10-32

18. Wang L, Feng L, Yao Y, Wang Y, Chen Y, Feng J, Xing Y: Optimal optic nerve sheath diameter threshold for the identification of elevated opening pressure on lumbar puncture in a Chinese population. PLoS One. 2015, 10:0117939. 10.1371/journal.pone.0117939

19. Bäuerle J, Lochner P, Kaps M, Nedelmann M: Intra- and interobsever reliability of sonographic assessment of the optic nerve sheath diameter in healthy adults. J Neuroimag. 2012, 22:42-45. 10.1111/j.15526569.2010.00546.x

20. Asghar A, Hashmi M, Hussain A: Optic nerve sheath diameter evaluated by transorbital sonography in healthy volunteers from Pakistan. Anaesth Pain Intensive Care. 2015, 19:282-286.

21. Hansen HC, Lagrèze W, Krueger O, Helmke K: Dependence of the optic nerve sheath diameter on acutely applied subarachnoidal pressure-an experimental ultrasound study. Acta Ophthalmol. 2011, 89:528-532. 10.1111/j.1755-3768.2011.02159.x

22. Launey Y, Nesseler N, Le Maguet P, Mallédant Y, Seguin P: Effect of osmotherapy on optic nerve sheath diameter in patients with increased intracranial pressure. J Neurotrauma. 2014, 31:984-988. 10.1089/neu.2012.2829

23. Killer HE, Laeng HR, Flammer J, Groscurth P: Architecture of arachnoid trabeculae, pillars, and septa in the subarachnoid space of the human optic nerve: anatomy and clinical considerations. Br J Ophthalmol. 2003, 87:777-781. 10.1136/bjo.87.6.777

24. Bäuerle J, Schuchardt F, Schroeder L, Egger K, Weigel M, Harloff A: Reproducibility and accuracy of optic nerve sheath diameter assessment using ultrasound compared to magnetic resonance imaging. BMC Neurol. 2013, 13:187. 10.1186/1471-2377-13-187

25. Shirodkar CG, Munta K, Rao SM, Mahesh MU: Correlation of measurement of optic nerve sheath diameter using ultrasound with magnetic resonance imaging. Indian J Crit Care Med. 2015, 19:466-470. $10.4103 / 0972-5229.162465$ 